\title{
Approximations of the boundary crossing probabilities for the maximum of moving weighted sums
}

\author{
Jack Noonan $^{1} \cdot$ Anatoly Zhigljavsky ${ }^{1}$
}

Received: 12 February 2018 / Revised: 5 May 2018 / Published online: 18 June 2018

(C) The Author(s) 2018

\begin{abstract}
We study approximations of boundary crossing probabilities for the maximum of moving weighted sums of i.i.d. random variables. We consider a particular case of weights obtained from a trapezoidal weight function which, under certain parameter choices, can also result in an unweighted sum. We demonstrate that the approximations based on classical results of extreme value theory provide some scope for improvement, particularly for a range of values required in practical applications.
\end{abstract}

Keywords Moving weighted sum $\cdot$ Boundary crossing probability $\cdot$ Moving weighted sum of squares $\cdot$ Change-point detection $\cdot$ Singular spectrum analysis

\section{Introduction: statement of the problem}

Let $\varepsilon_{1}, \varepsilon_{2}, \ldots$ be a sequence of independent identically distributed random variables with finite mean $\mu$ and variance $\sigma^{2}$ and some c.d.f. $F$. Define the moving weighted sum as

$$
\mathcal{S}_{n ; L, Q}=\sum_{s=n+1}^{n+L+Q-1} w_{L, Q}(s-n) \varepsilon_{s} \quad(n=0,1, \ldots)
$$

\footnotetext{
Jack Noonan

Noonanj1@cardiff.ac.uk

Anatoly Zhigljavsky

ZhigljavskyAA@cardiff.ac.uk

1 School of Mathematics, Cardiff University, Cardiff CF24 4AG, UK
} 
Fig. 1 The weight function $w_{L, Q}(\cdot), 1 \leq Q \leq L$

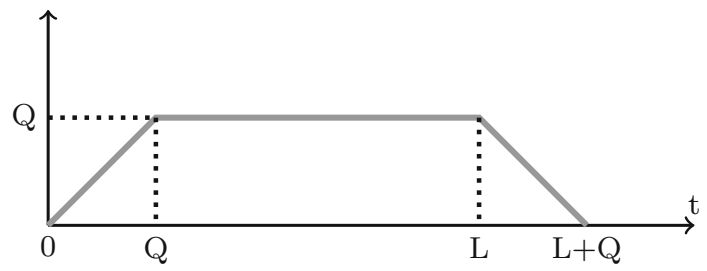

where the weight function $w_{L, Q}(\cdot)$ is defined by

$$
w_{L, Q}(t)= \begin{cases}t & \text { for } 0 \leq t \leq Q \\ Q & \text { for } Q \leq t \leq L \\ L+Q-t & \text { for } L \leq t \leq L+Q-1\end{cases}
$$

where $L$ and $Q$ are positive integers with $Q \leq L$.

The weight function $w_{L, Q}(\cdot)$ is depicted in Fig. 1 . In the special case $Q=1$, the weighted moving sum (1) becomes an ordinary moving sum.

The main aim of this paper is to study precision of different approximations of boundary crossing probabilities for the maximum of the moving weighted sum; that is,

$$
P\left(\max _{n=0,1, \ldots, M} \mathcal{S}_{n ; L, Q}>H\right)
$$

where $H$ is a given threshold, $M$ is reasonably large and $L, Q$ are fixed parameters.

This paper is structured as follows. In Sect. 2 we reformulate the problem and provide motivation why a trapezoidal weight function is considered. In Sect. 3, a number of approximations to (3) are introduced based on the classical extreme value theory. Using the classical approximations, which do not perform very well, we also derive another approximation (called 'combined') which appears to be more accurate. The performance of these approximations is analyzed by a large simulation study described in Sect. 4.

\section{Boundary crossing probabilities: discrete and continuous time}

\subsection{Reformulation of the problem}

For convenience of dealing with the probability (3), we standardise the moving weighted sum $\mathcal{S}_{n ; L, Q}$. Derivation of the following lemma is straightforward.

Lemma 1 The first two moments of $\mathcal{S}_{n ; L, Q}$ are

$$
E \mathcal{S}_{n ; L, Q}=\mu L Q, \quad \operatorname{var}\left(\mathcal{S}_{n ; L, Q}\right)=\frac{\sigma^{2} Q}{3}\left(3 L Q-Q^{2}+1\right)
$$


We now define the standardized random variables (r.v.)

$$
\zeta_{n}:=\frac{\mathcal{S}_{n ; L, Q}-E \mathcal{S}_{n ; L, Q}}{\sqrt{\operatorname{var}\left(\mathcal{S}_{n ; L, Q}\right)}}=\frac{\sqrt{3}\left(\mathcal{S}_{n ; L, Q}-\mu L Q\right)}{\sigma \sqrt{Q\left(3 L Q-Q^{2}+1\right)}},
$$

$n=0,1, \ldots$ If the r.v. $\varepsilon_{1}, \varepsilon_{2}, \ldots$ are normal then the r.v. $\zeta_{1}, \zeta_{2}, \ldots$ are also normal. Otherwise, using the Central Limit Theorem, we obtain that $\zeta_{n} \sim N(0,1)$ holds asymptotically, as $L \rightarrow \infty$.

Using the notation $\zeta_{n}$, our problem (3) is equivalent to studying approximations for the boundary crossing probability (abbreviated BCP)

$$
P_{M, h}\left(\zeta_{n}\right):=P\left(\max _{n=0,1, \ldots, M} \zeta_{n}>h\right),
$$

where

$$
H=\mu L Q+\sigma h \sqrt{\frac{Q\left(3 L Q-Q^{2}+1\right)}{3}} .
$$

A number of approaches could be used to approximate (6). We could have ignored the dependence structure of the sequence of moving weighted sums and used either asymptotic normality alone or the limiting extreme value distribution to choose $h$. Instead, in what follows we study several approximations of (6) which are based on approximating the sequence $\zeta_{n}$ by a continuous time random process. Before we proceed, let us consider a special case of $\varepsilon_{j}$, which has important practical significance.

\subsection{Motivation for the problem}

If we let $\varepsilon_{j}=\xi_{j}^{2}$, where $\xi_{1}, \xi_{2}, \ldots$ are i.i.d random variables with zero mean, variance $\delta^{2}$ and finite fourth moment $\mu_{4}=E \xi_{i}^{4}$, then $\mathcal{S}_{n ; L, Q}$ can be seen as a moving weighted sum of squares. In this case, the mean $\mu=E \varepsilon_{j}=\delta^{2}$ and $\sigma^{2}=\operatorname{var}\left(\varepsilon_{j}\right)=\mu_{4}-\delta^{4}$. By approximating (3) we are considering a particularly interesting case linked to the SSA change-point detection algorithm proposed in Moskvina and Zhigljavsky (2003). A good approximation for the BCP for the maximum of the moving weighted sums of squares is needed in the theory of sequential change-point detection because the BCP defines the significance levels for the SSA change-point detection statistic. For an extensive introduction to SSA, see Golyandina et al. (2001) and Golyandina and Zhigljavsky (2013).

\subsection{Continuous time approximation}

By the definition, the probability $P_{M, h}\left(\zeta_{n}\right)$ is an $(M+1)$-dimensional integral which is difficult to compute. We assume that $L \rightarrow \infty$ and consider a transformation described below in Sect. 3 from the time series $\zeta_{n}, n=0,1, \ldots, M$, to a continuous-time process 
$\zeta_{t}, t \in[0, T]$, where $T=M / \sqrt{L Q}$ for large $Q$, see (10), and $T=M / L$ in the case of small $Q$, see beginning of Sect. 3.2. Like the time series $\zeta_{n}$, the process $\zeta_{t}$ is standardized so that $E \zeta_{t}=0$ and $E \zeta_{t}^{2}=1$ for all $t$. Also, the process $\zeta_{t}$ is Gaussian and stationary with some autocorrelation function $R(s)=E \zeta_{0} \zeta_{s}$.

By such a transformation, the probability $P_{M, h}\left(\zeta_{n}\right)$ is approximated by $P\left(T, h, \zeta_{t}\right)$, which is the probability of reaching the threshold $h$ by the process $\zeta_{t}$ on the interval $[0, T]$; that is,

$$
\begin{aligned}
P_{M, h}\left(\zeta_{n}\right) & \cong P\left(T, h, \zeta_{t}\right)=\operatorname{Pr}\left\{\max _{0 \leq t \leq T} \zeta_{t} \geq h\right\} \\
& =\operatorname{Pr}\left\{\zeta_{t} \geq h \text { for at least one } t \in[0, T]\right\}
\end{aligned}
$$

For the continuous process $\zeta_{t}$, two main useful characteristics are the probability density function of reaching the threshold $h$ for the first time

$$
q\left(t, h, \zeta_{t}\right)=\frac{d}{d t} P\left(t, h, \zeta_{t}\right), \quad 0<t<\infty
$$

and the average time $\varrho\left(h, \zeta_{t}\right)$ until the process $\zeta_{t}$ reaches the threshold $h$

$$
E\left(\varrho\left(h, \zeta_{t}\right)\right)=\int_{0}^{\infty} t q\left(t, h, \zeta_{t}\right) d t=\int_{0}^{\infty} t d P\left(t, h, \zeta_{t}\right) .
$$

From the practical point of view, we are interested in finding good approximations of (6) for small and moderate $M$. But the mathematical theory guarantees accurate approximations just for large $M$.

To proceed further, we need to discuss results concerning the autocorrelation function of the continuous process $\zeta_{t}$. This can be done through computing the correlations between $\mathcal{S}_{n ; L, Q}$ and $\mathcal{S}_{n+v, L, Q}$ for $v>0$.

\subsection{Correlation between $\mathcal{S}_{n ; L, Q}$ and $\mathcal{S}_{n+1 ; L, Q}$}

For fixed $L$ and $Q$, the moving weighted sum $\mathcal{S}_{n ; L, Q}$ is a function of $n$. The index $n$ can be treated as time and thus the sequence $\mathcal{S}_{0 ; L, Q}, \mathcal{S}_{1 ; L, Q}, \ldots$ defined in (1) can be considered as a time series. In order to derive our approximations, we need explicit expressions for the correlation $\operatorname{Corr}\left(\mathcal{S}_{n ; L, Q}, \mathcal{S}_{n+1 ; L, Q}\right)$. The general case $\operatorname{Corr}\left(\mathcal{S}_{n ; L, Q}, \mathcal{S}_{n+v ; L, Q}\right), v>1$ need not be considered for these approximations.

Without loss of generality, we can assume that $n=0$ and we denote $\mathcal{S}_{v}:=\mathcal{S}_{v, L, Q}$ where $v=0,1$.

Lemma 2 The correlation $\operatorname{Corr}\left(\mathcal{S}_{0}, \mathcal{S}_{1}\right)=\operatorname{Corr}\left(\mathcal{S}_{n ; L, Q}, \mathcal{S}_{n+1 ; L, Q}\right)$, where $\mathcal{S}_{n ; L, Q}$ is defined in (1), is

$$
\operatorname{Corr}\left(\mathcal{S}_{0}, \mathcal{S}_{1}\right)=\frac{E\left(\mathcal{S}_{0} \mathcal{S}_{1}\right)-\left(E \mathcal{S}_{0}\right)^{2}}{\operatorname{var}\left(\mathcal{S}_{0}\right)}=1-\frac{3}{3 L Q-Q^{2}+1} .
$$


Proof From the definition (1), the quadratic forms $\mathcal{S}_{0}$ and $\mathcal{S}_{1}$ can be represented as

$$
\mathcal{S}_{0}=\sum_{i=1}^{Q-1} i \varepsilon_{i}+Q \sum_{i=Q}^{L} \varepsilon_{i}+\sum_{i=L+1}^{Q+L-1}(Q+L-i) \varepsilon_{i}
$$

and

$$
\mathcal{S}_{1}=\mathcal{S}_{0}-\sum_{i=1}^{Q} \varepsilon_{i}+\sum_{i=1}^{Q} \varepsilon_{L+i}
$$

Using these representations, we can easily obtain $E\left(\mathcal{S}_{0} \mathcal{S}_{1}\right)=E \mathcal{S}_{0}^{2}-Q \sigma^{2}$. Then by substituting the explicit expressions (4) for $E \mathcal{S}_{0}$ and $\operatorname{var}\left(\mathcal{S}_{0}\right)=E \mathcal{S}_{0}^{2}$, we obtain the desired result.

Note that the correlation does not depend on the distribution of errors $\varepsilon_{j}$ (unlike the covariance which depends on the mean $\mu$ and variance $\sigma^{2}$ of $\varepsilon_{j}$ ). This also can be seen in relation to the fact (see, for example, Priestley 1981) that the spectral density of the moving average process depends only on the weight function, which is $w_{L, Q}(t)$ in our case.

\section{Approximations of the boundary crossing probabilities}

In this section we formulate four different approximations for the $\mathrm{BCP} P_{M, h}\left(\zeta_{n}\right)$ defined in (7). These approximations depend on the behaviour of the autocorrelation function $R(s)=E \zeta_{0} \zeta_{s}$ at 0 which in its turn depends on parameters $Q$ and $L$ of the weight function in (2). We consider the following two cases: (i) large $Q$ and large $L$, (ii) small $Q$ and large $L$.

\subsection{Case of large $Q$ and large $L$}

Consider the sequence of random variables $\zeta_{0}, \zeta_{1}, \ldots, \zeta_{M}$ defined in (5). In view of Lemma 2, the correlation between $\zeta_{n}$ and $\zeta_{n+1}$ is

$$
\operatorname{Corr}\left(\zeta_{n}, \zeta_{n+1}\right)=1-\frac{3}{3 L Q-Q^{2}+1}
$$

Assume that both $L$ and $Q$ are large. Moreover, assume that $L$ and $Q$ tend to infinity in such a way that the limit $\lambda=\lim Q / L$ exists and $0<\lambda \leq 1$. Set $\Delta=1 / \sqrt{L Q}$ and

$$
t_{n}=n \Delta, \quad n=0,1, \ldots, M, \quad \text { so that } t_{n} \in[0, T] \text { with } T=M \Delta .
$$


Define a piece-wise linear continuous-time process $\zeta_{t}^{(L)}, t \in[0, T]$, as follows

$$
\zeta_{t}^{(L)}=\frac{1}{\Delta}\left[\left(t_{n}-t\right) \zeta_{n-1}+\left(t-t_{n-1}\right) \zeta_{n}\right] \text { for } t \in\left[t_{n-1}, t_{n}\right], n=1, \ldots, M
$$

By construction, the process $\zeta_{t}^{(L)}$ is such that $\zeta_{t_{n}}^{(L)}=\zeta_{n}$ for $n=0, \ldots, M$. Also we have that $\zeta_{t}^{(L)}$ is a second-order stationary process in the sense that $E \zeta_{t}^{(L)}, \operatorname{var}\left(\zeta_{t}^{(L)}\right)$ and the autocorrelation function $R_{\zeta}^{(L)}(t, t+k \Delta)=\operatorname{Corr}\left(\zeta_{t}^{(L)}, \zeta_{t+k \Delta}^{(L)}\right)$ do not depend on $t$.

Lemma 3 Let $\lambda=\lim _{L, Q \rightarrow \infty} Q /$ L and assume that $0<\lambda \leq 1$. Consider the process $\zeta_{t}^{(L)}$ defined in (11). The limiting process $\zeta_{t}=\lim _{L, Q \rightarrow \infty} \zeta_{t}^{(L)}$ is stationary Gaussian with some autocorrelation function $R_{\zeta}(t, t+s)=R(s)$. Moreover, $R^{\prime}(0)=0$ and $R^{\prime \prime}(0)=-6 /(3-\lambda)$.

Proof For the autocorrelation function $R(\cdot)$ we have $R^{\prime}(0)=0$ since

$$
R^{\prime}(0-)=R^{\prime}(0+)=\lim _{L, Q \rightarrow \infty} \frac{R(\Delta)-1}{\Delta}=\lim _{L, Q \rightarrow \infty} \frac{-3 \sqrt{L Q}}{3 L Q-Q^{2}+1}=0
$$

where we used the relations $\Delta=1 / \sqrt{L Q}, R(\Delta)=1-3 /\left(3 L Q-Q^{2}+1\right)$ and $R(0)=1$. We similarly obtain

$$
\begin{aligned}
R^{\prime \prime}(0) & =\lim _{L, Q \rightarrow \infty} \frac{R(\Delta)+R(-\Delta)-2 R(0)}{\Delta^{2}}=\lim _{L, Q \rightarrow \infty} \frac{-6 L Q}{3 L Q-Q^{2}+1} \\
& =-\frac{6}{3-\lambda}<0 .
\end{aligned}
$$

For a Gaussian stationary process $\zeta_{t}$ with $E \zeta_{t}=0$ and $E \zeta_{t}^{2}=1$ and autocorrelation function $R(\cdot)$ such that $R^{\prime}(0)=0$ and $R^{\prime \prime}(0)<0$ we can use the following two well-known approximations.

Approximation 1 (App 1) From Theorem 8.2.7 in Leadbetter et al. (1983) we have

$$
\lim _{T \rightarrow \infty} P\{\max _{0 \leq t \leq T} \zeta_{t} \leq \underbrace{\frac{u+\log \frac{\sqrt{-R^{\prime \prime}(0)}}{2 \pi}}{\sqrt{2 \log T}}+\sqrt{2 \log T}}_{h}\}=\exp \left(-e^{-u}\right) .
$$

Expressing $u$ in terms of $h$, we obtain the Approximation 1

$$
P\left(T, h, \zeta_{t}\right) \cong 1-\exp \left(-e^{-u}\right)
$$


with $u=\gamma(h-\gamma)+c$, where

$$
\gamma=\sqrt{2 \log T} \text { and } c=-\log \frac{\sqrt{-R^{\prime \prime}(0)}}{2 \pi}=-\log \frac{1}{2 \pi} \sqrt{\frac{6}{3-\lambda}} .
$$

Approximation 2 (App 2) From Cramér (1965), we have

$$
\lim _{T \rightarrow \infty} P\{\max _{0 \leq t \leq T} \zeta_{t} \leq \underbrace{\sqrt{2 \log \mu}+\frac{v}{\sqrt{2 \log \mu}}}_{h}\}=\exp \left(-e^{-v}\right),
$$

where

$$
\mu=\frac{T \sqrt{-R^{\prime \prime}(0)}}{2 \pi}=\frac{T}{2 \pi} \sqrt{\frac{6}{3-\lambda}} .
$$

Expressing $v$ in terms of $h$, we obtain Approximation 2

$$
P\left(T, h, \zeta_{t}\right) \cong 1-\exp \left(-e^{-v}\right)
$$

with

$$
v=\sqrt{2 \log \mu}(h-\sqrt{2 \log \mu}) .
$$

Note that $2 \log \mu=\gamma^{2}-2 c$ and

$$
\sqrt{2 \log \mu}=\sqrt{\gamma^{2}-2 c}=\gamma-\frac{c}{\gamma}+O\left(\frac{1}{\gamma^{3}}\right),
$$

as $\gamma \rightarrow \infty$, where $\gamma$ and $c$ are defined in (13). Therefore, for large $T$ (and, therefore, large $\gamma$ ) we have

$$
v \cong\left(\gamma-\frac{c}{\gamma}\right)\left(h-\gamma+\frac{c}{\gamma}\right)=\underbrace{(h-\gamma) \gamma+c}_{u}-\frac{(h-\gamma) c}{\gamma}-\frac{c^{2}}{\gamma^{2}} .
$$

Let us construct another approximation by combining the Approximations 1 and 2. Approximation 3 (Combined) Consider the approximation

$$
P\left(T, h, \zeta_{t}\right) \cong 1-\exp \left(-e^{-z}\right)
$$

where

$$
z= \begin{cases}u-\frac{(h-\gamma) c}{\gamma}-\frac{c^{2}}{\gamma^{2}} & \text { for } \quad h \leq \gamma-\frac{c}{\gamma} \\ u & \text { for } h \geq \gamma-\frac{c}{\gamma}\end{cases}
$$


Formally, $\lambda=\lim _{L, Q \rightarrow \infty} Q / L=0$ still satisfies Lemma 3 in the sense that $R^{\prime}(0)=0$ and $R^{\prime \prime}(0)=-2<0$; however, the above approximations are poor when $Q$ is small; this shall be demonstrated in Sect. 4. The case of small $Q$ should be treated differently and is considered in the following subsection.

\subsection{Case of small $Q$ and large $L$}

Consider again the sequence of random variables $\zeta_{n}$ defined by (5). Unlike in Sect. 3.1, now we look at the asymptotic transformation when $L \rightarrow \infty$ but $Q$ is fixed. Set $\Delta=1 / L$ and $T=M \Delta$. Define $t_{n}, n=0,1, \ldots, M$, as in (10) and consider the piece-wise linear continuous-time process $\zeta_{t}^{(L)}$ defined by (11).

Lemma 4 Let $Q$ be fixed. The limiting process $\zeta_{t}$ as $L \rightarrow \infty$ is a Gaussian secondorder stationary process with autocorrelation function $R_{\zeta}(t, t+s)=R(s)$. Moreover, $R^{\prime}(0+)=-\frac{1}{Q} \neq 0$.

Proof We first note that

$$
\left.\frac{\partial R_{\zeta}(t, s)}{\partial s}\right|_{s=t+}=R(0+)
$$

Using (9) and the fact that $\Delta=1 / L$, we have

$$
R^{\prime}(0+)=\lim _{L \rightarrow \infty} \frac{R(\Delta)-R(0)}{\Delta}=-\lim _{L \rightarrow \infty} \frac{3 L}{3 L Q-Q^{2}+1}=-\frac{1}{Q}
$$

Let us now formulate the tangent approximation suggested in Durbin (1985); it is one of the most known approximations for the density function $q\left(t, h, \zeta_{t}\right)$ of the first passage time defined in (8). Using this, we can approximate the first passage probability $P\left(T, h, \zeta_{t}\right)$ defined in (7) in the case of a Gaussian process $\zeta(t)$ on $[0, T]$ with $E \zeta(t)=0$, some autocorrelation function $R_{\zeta}(t, s)$ and the possibly non-constant threshold $h=h(t)$.

The Durbin approximation for $q\left(t, h, \zeta_{t}\right)$ can be written as

$$
q\left(t, h, \zeta_{t}\right) \cong b_{0}(t, h) f(t, h)
$$

where

$$
f(t, h)=\frac{1}{\sqrt{2 \pi R_{\zeta}(t, t)}} e^{-\frac{h^{2}(t)}{2 R_{\zeta}(t, t)}} b_{0}(t, h)=-\left.\frac{h(t)}{R_{\zeta}(t, t)} \frac{\partial R_{\zeta}(s, t)}{\partial s}\right|_{s=t+}-\frac{d h(t)}{d t} .
$$

In view of (8) the related approximation for the first passage probability $P\left(T, h, \zeta_{t}\right)$ is

$$
P\left(T, h, \zeta_{t}\right) \cong \int_{0}^{T} b_{0}(t, h) f(t, h) d t
$$


In the case when the threshold $h(t)=h$ is constant, using Lemma 4 we obtain

$$
b_{0}(t, h)=-h R^{\prime}(0+)=\frac{h}{Q}, \quad q\left(t, h, \zeta_{t}\right) \cong \frac{h}{\sqrt{2 \pi} Q} e^{-h^{2} / 2}
$$

and therefore we obtain the following approximation.

Approximation 4 (App 4) The Durbin approximation for the BCP (7) is

$$
P\left(T, h, \zeta_{t}\right) \cong \frac{h T}{\sqrt{2 \pi} Q} e^{-h^{2} / 2}
$$

\section{Simulation study}

In this section we study quality of approximations for the BCP $P_{M, h}\left(\zeta_{n}\right)$ defined in (6), where $\varepsilon_{t}$ are normal r.v.'s with mean 0 and variance 1 . Asymptotically (for large $L$ and $M$ ), the approximations we study can also be used for the BCP connected to the weighted sum of squares discussed in Sect. 2.2 and therefore for setting significance levels for the SSA change-point statistic defined in Moskvina and Zhigljavsky (2003).

In Figs. 2, 3, 4, 5, and 6, the 'Sum of normal' line corresponds to the empirical value of (6) computed from 100,000 simulations with different values of $L, Q$ and $M$. In simulations leading to Figs. 2, 3, and 4 the value of $Q$ can be considered as large and hence we compare Approximations 1-3. In Fig. 5 we present analysis demonstrating the lack of accuracy of Approximations 1-3 when $Q$ is small. We then analyse the performance of the Durbin approximation in Fig. 6, which is constructed specifically under the assumption that $Q$ is small; in this case we set $Q=1$. We observe that for large $L$ and $Q$ Approximation 3 is typically superior to the Approximations 1 and 2 for all $h$ (note that Approximations 1 and 3 coincide for large values of $h$ ). Listed in Tables 1, 2, 3, and 4 are the approximated threshold values $h$ (for Approximations 1 and 2 only) for a specified true BCP, when this BCP is small enough. In these tables, R.E. denotes the relative error.

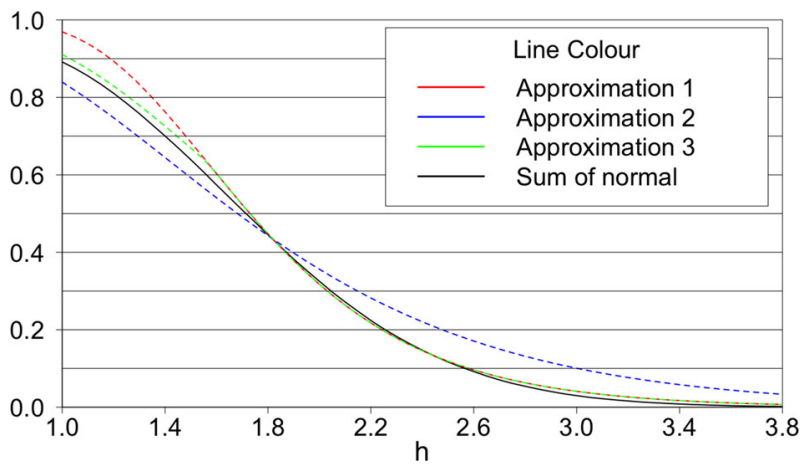

Fig. 2 The BCP for the weighted sum of normal r.v. and its approximations: $L=150, Q=50, M=1000$. (Color figure online) 


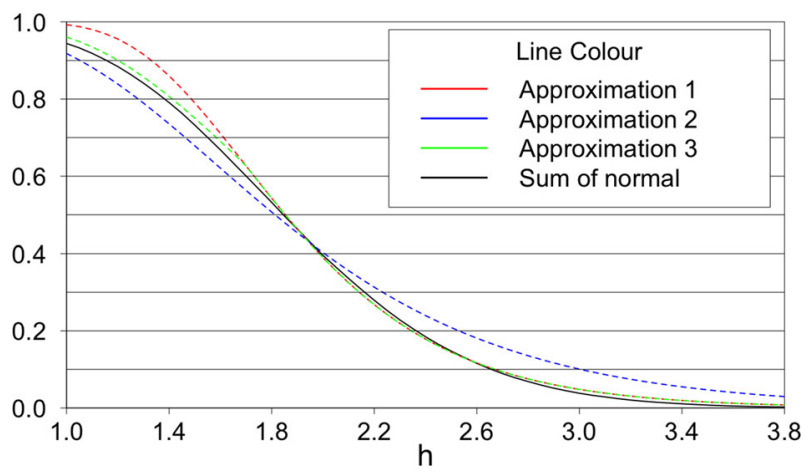

Fig. 3 The BCP for the weighted sum of normal r.v. and approximations: $L=100, Q=50, M=1000$. (Color figure online)

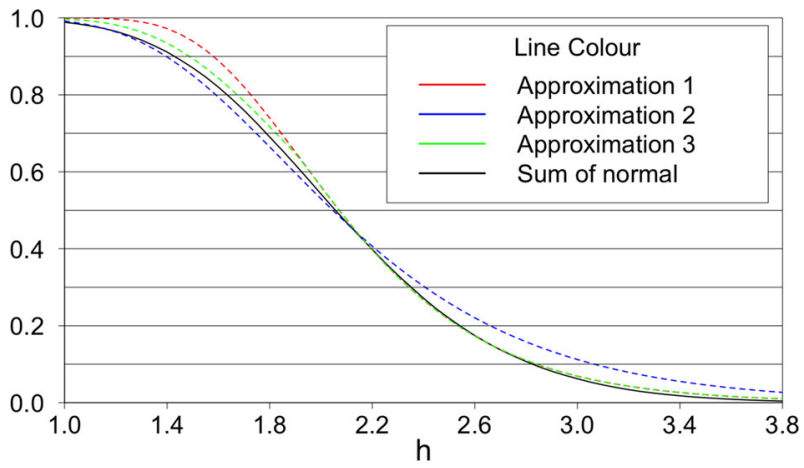

Fig. 4 The BCP for the weighted sum of normal r.v. and its approximations: $L=100, Q=100, M=$ 2000. (Color figure online)

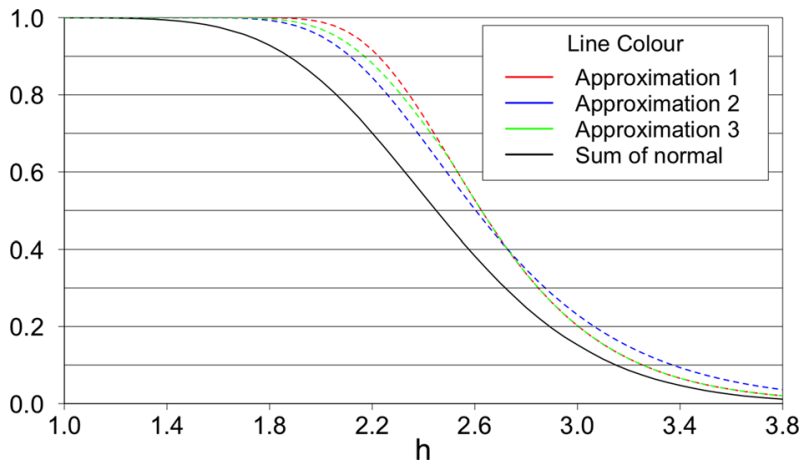

Fig. 5 The BCP for the weighted sum of normal r.v. and its approximations: $L=100, Q=5, M=2000$. (Color figure online) 

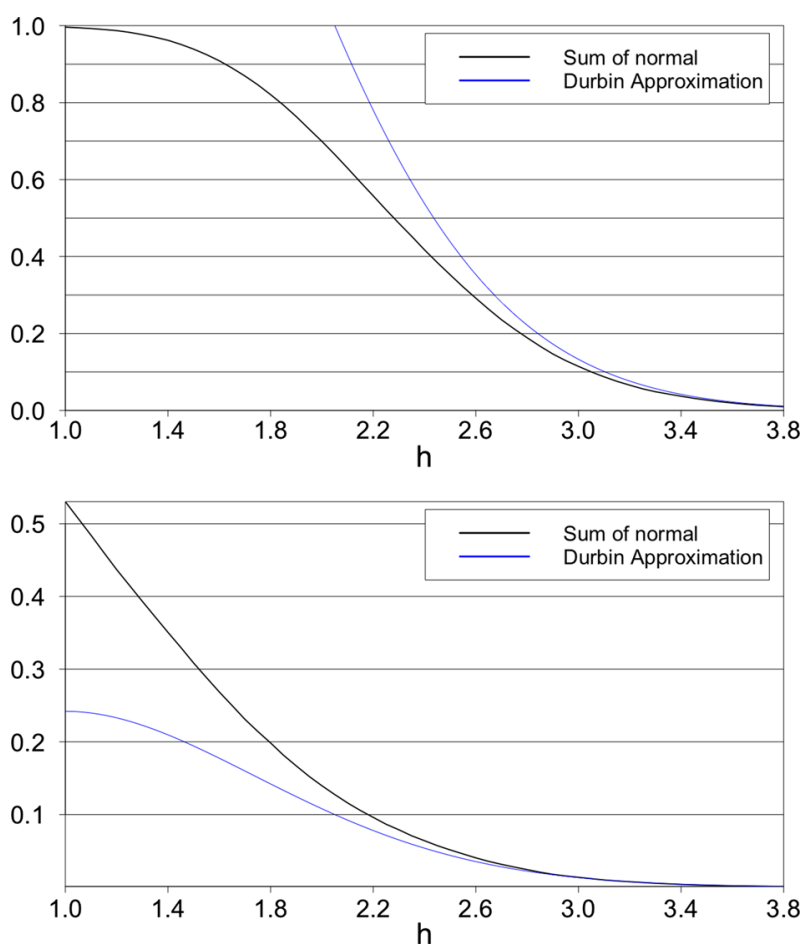

Fig. 6 The BCP for the weighted sum of normal random variables and the Durbin approximation: $L=300, Q=1, T=10$ (top) and $L=300, Q=1, T=1$ (bottom). (Color figure online)

Table 1 Threshold for a given BCP for the weighted sum of normal r.v. and approximations: $L=150$, $Q=50, M=1000$

\begin{tabular}{llllll}
\hline BCP & Sum of normal & App 1 & App 2 & R.E. for App 1 (\%) & R.E. for App 2 (\%) \\
\hline 0.05 & 2.833 & 2.907 & 3.510 & 2.612 & 23.897 \\
0.10 & 2.572 & 2.582 & 3.004 & 0.389 & 16.796 \\
0.15 & 2.401 & 2.386 & 2.700 & 0.625 & 12.453 \\
0.20 & 2.264 & 2.243 & 2.477 & 0.928 & 9.408 \\
\hline
\end{tabular}

As seen in Fig. 2 and Table 1, for the chosen parameters Approximation 2 is generally poor; for small BCP we see particularly high relative errors in Table 1 . On the other hand, Approximation 1 performs well for small BCP and, although discrepancies can be seen for small $h$, we see that Approximation 3 performs quite well across all values of $h$.

As shown in Fig. 3 and Table 2, Approximation 2, whilst still being considerably worse than Approximations 1 and 3, shows signs of improvement with this choice of $L$ and $Q$. At the BCP of 0.05 , Approximation 1 produces the lowest relative error with the parameter choices considered so far. 
Table 2 Threshold for a given BCP for the weighted sum of normal r.v. and its approximations: $L=100$, $Q=50, M=1000$

\begin{tabular}{lllllc}
\hline BCP & Sum of normal & App 1 & App 2 & R.E. for App 1 (\%) & R.E. for App 2 (\%) \\
\hline 0.05 & 2.911 & 2.984 & 3.460 & 2.508 & 18.859 \\
0.10 & 2.654 & 2.671 & 3.004 & 0.641 & 13.188 \\
0.15 & 2.491 & 2.483 & 2.730 & 0.321 & 9.595 \\
0.20 & 2.362 & 2.345 & 2.530 & 0.720 & 7.113 \\
\hline
\end{tabular}

Table 3 Threshold for a given BCP for the weighted sum of normal r.v. and approximations: $L=100$, $Q=100, M=2000$

\begin{tabular}{lllllc}
\hline BCP & Sum of normal & App 1 & App 2 & R.E. for App 1 (\%) & R.E. for App 2 (\%) \\
\hline 0.05 & 3.063 & 3.135 & 3.455 & 2.351 & 12.798 \\
0.10 & 2.816 & 2.841 & 3.066 & 0.888 & 8.878 \\
0.15 & 2.659 & 2.664 & 2.831 & 0.188 & 6.469 \\
0.20 & 2.541 & 2.534 & 2.660 & 0.275 & 4.683 \\
\hline
\end{tabular}

Table 4 Threshold for a given BCP for the weighted sum of normal r.v. and approximations: $L=100$, $Q=5, M=2000$

\begin{tabular}{llllll}
\hline BCP & Sum of normal & App 1 & App 2 & R.E. for App 1 (\%) & R.E. for App 2 (\%) \\
\hline 0.05 & 3.380 & 3.494 & 3.664 & 3.373 & 8.402 \\
0.10 & 3.150 & 3.254 & 3.371 & 3.302 & 7.016 \\
0.15 & 3.007 & 3.109 & 3.194 & 3.392 & 6.219 \\
0.20 & 2.896 & 3.004 & 3.065 & 3.729 & 5.836 \\
\hline
\end{tabular}

As shown in Fig. 4 and Table 3, we see a considerable improvement in Approximation 2 with the increase in $M$ from 1000 to 2000, however Approximation 3 still remains far superior. For this larger $M$, Approximation 1 shows the smallest relative error at a BCP of 0.05 which is arguably the most important case.

We shall now consider the performance of Approximations 1-3 for small $Q$. We conclude that all three approximations perform poorly when $Q$ is not large enough (of order $L$ ).

As can be seen from Fig. 5 and Table 4, all three approximations are poor for $Q=5$. Relative errors are high and thus the use of these approximations for the case of small $Q$ and large $L$ cannot be justified.

For checking the quality of the Durbin approximation we used the same settings as for the Approximations 1, 2 and 3. In Fig. 6, we show results for the Durbin approximation for a few particular values of $L$ and $Q$.

We can conclude that the quality of the Durbin approximation (16) is poor unless the threshold $h$ is very large. This is seen graphically in Fig. 6 as well as numerically in Table 5, where there is a sharp increase in the relative error as the BCP increases. 
Table 5 Threshold for a given BCP for the weighted sum of normal r.v. and Durbin approximation: $L=300, Q=1, T=1$

\begin{tabular}{llll}
\hline BCP & Sum of normal & Durbin approx. & R.E. for Durbin approx. (\%) \\
\hline 0.05 & 2.520 & 2.436 & 3.333 \\
0.10 & 2.190 & 2.049 & 6.438 \\
0.15 & 1.970 & 1.756 & 10.863 \\
0.20 & 1.794 & 1.464 & 18.395 \\
\hline
\end{tabular}

For the BCP of 0.05 the relative error for the Durbin approximation is higher than all relative errors of Approximation 1 considered in this paper.

\section{Conclusion}

A number of approximations of boundary crossing probabilities for the maximum of moving weighted sums of i.i.d. random variables have been considered. The particular weights are obtained from a trapezoidal weight function that has important links to the SSA change-point detection algorithm described in Moskvina and Zhigljavsky (2003). We have seen that Approximations 1-3 perform rather well for large $Q$ and $L$, and Approximation 3 consistently outperforming Approximations 1 and 2 across all values of the threshold $h$. The case of small $Q$ must be considered separately since Approximations 1-3 perform poorly. The Durbin approximation, developed for small $Q$, is not satisfactory, unless threshold $h$ is very large.

Acknowledgements The authors are grateful to both referees for their constructive comments.

Open Access This article is distributed under the terms of the Creative Commons Attribution 4.0 International License (http://creativecommons.org/licenses/by/4.0/), which permits unrestricted use, distribution, and reproduction in any medium, provided you give appropriate credit to the original author(s) and the source, provide a link to the Creative Commons license, and indicate if changes were made.

\section{References}

Cramér H (1965) A limit theorem for the maximum values of certain stochastic processes. Theory Probab Appl 10(1):126-128

Durbin J (1985) The first-passage density of a continuous Gaussian process to a general boundary. J Appl Probab 22:99-122

Golyandina N, Zhigljavsky A (2013) Singular spectrum analysis for time series. Springer briefs in statistics. Springer, Berlin

Golyandina N, Nekrutkin V, Zhigljavsky AA (2001) Analysis of time series structure: SSA and related techniques, monographs on statistics and applied probability, vol 90. Chapman \& Hall, London

Leadbetter MR, Lindgren G, Rootzén H (1983) Extremes and related properties of random sequences and processes, vol 21. Springer, New York

Moskvina V, Zhigljavsky A (2003) An algorithm based on singular spectrum analysis for change-point detection. Commun Stat 32(2):319-352

Priestley MB (1981) Spectral analysis and time series. Academic Press, London 